\title{
Encouraging Task-Related Dialog in 2D and 3D Shared Narrative Workspaces
}

\author{
Karl E. Steiner \\ University of North Texas \\ General Academic Building, Room 320 \\ Denton, Texas 76203 \\ steiner@cs.unt.edu
}

\author{
Tom Moher \\ University of Illinois at Chicago \\ 851 S. Morgan (M/C 152), Room 1120 SEO \\ Chicago, IL 60607-7053 \\ moher@uic.edu
}

\begin{abstract}
This paper describes a series of studies conducted to evaluate methods of encouraging productive dialog between young children in 2D and 3D shared narrative workspaces. These workspaces were based on the Graphic Story Writer (GSW), an interactive system that produces coherent narrative text in response to user interactions. In particular, we examine the effect of introducing "positive interdependence" between collaborators as a way of encouraging productive dialog.

Three studies of user interactions with the GSW were performed. An initial study of collaborative storytelling using a 2D version of the GSW demonstrated the technical feasibility of creating a shared 2D workspace devoted to narrative construction The study also confirmed the difficulty of encouraging communication between children during synchronous learning activities at separate computers. The second study introduced an additional condition that created positive interdependence between users. Users in this condition had significantly more on-task communication than those in the other conditions. We go on to describe how we modified the 2D GSW model to support collaboration in a 3D shared workspace, and provide observations and insights gained from a usability study of the 3D GSW.
\end{abstract}

\section{Categories and Subject Descriptors}

D.3.3 [Information Interfaces and Presentation]: User Interfaces - Graphical User Interfaces, Interaction Styles.

\section{General Terms}

Design, Human Factors

\section{Keywords}

Computer Supported Cooperative Work (CSCW), Shared Workspaces, Computer Supported Collaborative Learning (CSCL), Children, Narrative.

Permission to make digital or hard copies of all or part of this work for personal or classroom use is granted without fee provided that copies are not made or distributed for profit or commercial advantage and that copies bear this notice and the full citation on the first page. To copy otherwise, or republish, to post on servers or to redistribute to lists, requires prior specific permission and/or a fee.

CVE'02, September 30-October 2, 2002, Bonn, Germany.

Copyright 2002 ACM 1-58113-489-4/02/0009...\$5.00.

\section{INTRODUCTION}

A growing body of work points to the educational benefits of collaborative work $[5,9,15,16,17]$. Students in collaborative groups can discuss their work and present information in alternative forms. They can analyze the work of their partners in a more complete manner. Collaborative work also allows opportunities for peer modeling. Students can imitate the successful strategies of their peers. Collaborative work can enhance students' mood state, as well as increase their motivation and productivity. The idea that we can learn from dialog with others is also consistent with a constructivist view of learning [4] - that is, that learning is "an active, constructive, cumulative, selfregulated and goal-oriented process in which the learner plays a critical role [11]."

However, there is evidence of some barriers that can make collaborative work between young students in synchronous shared workspaces difficult. While a successful collaborative experience requires substantive dialogs between users in order to facilitate knowledge construction, discussions of this type will not necessarily occur spontaneously. In one study of shared workspaces, users reported that the use of side-by-side or remote workstations (rather than a single shared workstation) made shared understanding of the workspace more difficult, and was viewed as more difficult to use [10]. In observations of a collaborative virtual environment for children, researchers reported that the young users had difficulties recognizing shared goals and cooperating on them [7]. In a study of the use of storytelling technologies in the classroom, children were frequently observed to compete, to decline to contribute, and that for some, collaboration was difficult [3]. Simply placing students together at networked computers does not necessarily create a cooperative learning situation.

One possible technique for structuring activities in order to encourage collaboration is to establish positive interdependences between group members. Positive interdependence can be characterized as a situation where group members must rely on collaborators to provide resources or to fulfill roles in order to accomplish their task. While introducing complexities into a task is generally inconsistent with user-centered design, such "problematization" can be beneficial in collaborative learning [6].

\section{GRAPHIC STORY WRITER}

Storytelling provides an attractive domain in which to explore ideas for collaborative activities between children (including the establishment of positive interdependence). Storytelling is an important communication skill and provides opportunities for active engagement on the part of both the teller and the listener [2, 
14]. A number of recent CSCW and CSCL systems have explored the domain of storytelling $[1,3,7,8]$.

The collaborative environments used for the studies described in this paper were based on the Graphic Story Writer [12, 13]. The Graphic Story Writer (GSW) is an interactive learning environment for story construction, designed to address the difficulties young users face in story composition. The GSW gets its inspiration from story dictation, a technique used to support young students in the creation of complete stories. In the GSW, the user guides higher level plot structures through character interactions, while the system provides low level text structures. In this way, the GSW can provide support for young students as they build their own models of story structures. The original single-user version of the GSW was extended to support multiple users so that the important notion of storytelling as an act of communication and shared experience could be reinforced through collaboration with other students. Also, through collaboration, students would have the opportunity to engage in rich and genuine verbal interactions.

The basic interaction cycle of the GSW involves the user selecting a character or prop and moving it within the work area. Story text is then generated based on the attributes of the character or prop, prior story events, and the proximity to other objects within the workspace. For the evaluations described in this paper, the GSW was configured to use pre-selected sets of characters, props, and conflicts, though the system can also be configured to allow users to make these selections.

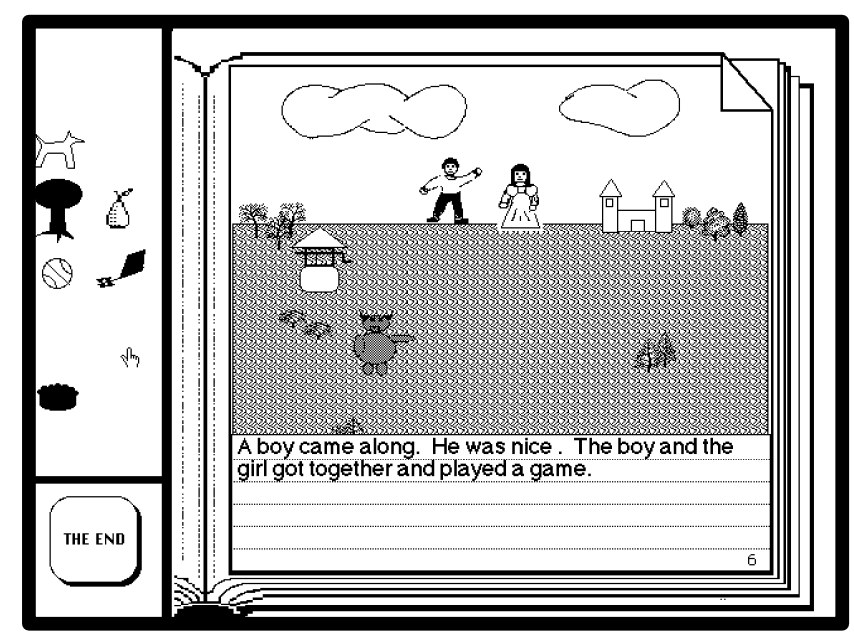

Figure 1: 2D Graphic Story Writer

\section{2D GSW INTERACTION STUDY 1}

In our work with the GSW, we had observed a strong social component to user interactions with the system. We felt that we could capitalize on students' desires to share their stories by creating a collaborative version of the GSW. We hoped to overcome some of the barriers common to shared workspaces and promote task-related verbal interactions between our young users.

We designed a multi-user version of the GSW to address the difficulties young users face in collaborative composition. In addition to supporting young students in the creation of complete stories, a collaborative version of the GSW would reinforce the important notion of storytelling as an act of communication and shared experience. Also, through collaboration, students would have the opportunity to engage in rich and genuine verbal interactions. They could ask and answer questions, be exposed to alternative viewpoints, and observe the problem solving strategies of their peers.

We hypothesized that by having the system handle lower level text structures, the GSW would allow users to devote more attention and discussion to the higher level issues of jointly constructing character interactions and story structures. In order to get initial feedback on the efficacy of a shared workspace in encouraging verbal interactions, we arranged a pilot study.

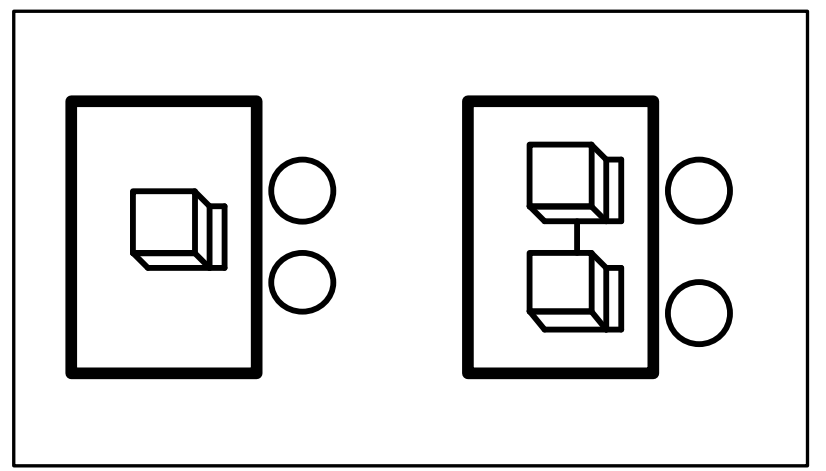

Figure 2: CO-PRESENT and REMOTE Workspaces

\subsection{Method}

Fourteen kindergarten and first-grade students participated in the study. The students were randomly assigned to pairs, which were then randomly assigned to one of two treatment groups. In the first group (COPRESENT), pairs of students shared a single computer running the GSW. In the other group (REMOTE), pairs of students worked on a shared workspace running simultaneously on two networked computers (Figure 9). Users in the REMOTE case each started their stories with similar objects in their staging area. Each individual was presented with two characters, one food item one place item and one toy item. This uniform distribution was chosen to present an environment consistent with that used by the COPRESENT groups.

The children were briefly trained in the use of the mouse and the operation of the Graphic Story Writer. They were then instructed to collaborate on a story and given ten minutes to work with the GSW. Audio and video records of the subjects' performances were collected, and transcripts of the verbal interactions (one per subject) were composed.

The transcripts were coded according to the types of utterances each subject produced:

- Declarations announcing proposed actions (e.g. I'm going to make the boy go behind the tree.)

- Intentions describing the user's motivation for an action (e.g. I want him to get away from the mean dog.)

- Observations of system events (e.g. The boy hid behind the tree.)

- Questions about the system events or user motivation (e.g. Why did he hide?)

- $\quad$ Simple Answers to direct questions about user actions (e.g. I moved him by the tree.) 
- $\quad$ Explanations of the cause behind system interpretations (e.g. He's hiding because he's shy.)

- Mechanics of system usage and coordination (e.g. Let me move the dog.)

The transcripts were coded by two independent scorers with strong agreement; the few discrepancies were resolved through negotiation. A record of the resulting stories was analyzed for conflict resolution structure.

\subsection{Results}

\begin{tabular}{|l|l|l|l|l|}
\hline Category & $\begin{array}{l}\text { Mean } \\
\text { REMOTE }\end{array}$ & $\begin{array}{l}\text { Mean } \\
\text { COPRESENT }\end{array}$ & $\boldsymbol{F} \mathbf{1 , 1 2}$ & P \\
\hline Question & 0.63 & 4.83 & 8.21 & .014 \\
\hline Answer & 0.50 & 4.00 & 5.60 & .036 \\
\hline Observation & 1.50 & 2.00 & 0.29 & .603 \\
\hline Explanation & 0.00 & 0.67 & 13.71 & .003 \\
\hline Suggestion & 0.38 & 11.83 & 6.73 & .024 \\
\hline Intention & 1.13 & 3.67 & 3.08 & .105 \\
\hline Mechanics & 0.00 & 2.17 & 2.04 & .179 \\
\hline Miscellaneous & 2.00 & 2.67 & 0.15 & .707 \\
\hline Total & 6.13 & 31.83 & 18.30 & .001 \\
\hline & & & & \\
\hline Completion & 0.25 & 0.67 & 2.52 & 0.14 \\
\hline
\end{tabular}

Table 1: Pilot Study Results

A single-factor (COPRESENT vs. REMOTE) analysis of variance was performed on the coded transcript data employing individual utterance types and utterance total as dependent variables, and on the resultant stories. The mean values and ANOVA results are listed in Table 6 .

While the mean number of stories embodying a complete conflict resolution structure was higher for the COPRESENT group, 0.67, as opposed to 0.25 for the REMOTE group, the difference was not statistically significant; $F(2,12)=2.52, \mathrm{p}=0.14$.

There was, however, a clear difference in the verbal interaction of the two groups. Over a ten-minute period, students in the REMOTE group averaged only about six utterances, compared to almost 32 for their counterparts in the COPRESENT group. This result is significant at $\mathrm{p}<.01 ; \mathrm{F}(2,12)=18.30, \mathrm{p}=0.001$. While members of the REMOTE group had fewer utterances than literal subjects in all categories, significant differences were found at $\mathrm{p}<$ .05 in the frequency of questions $(\mathrm{p}=0.014)$, answers $(\mathrm{p}=0.036)$, explanations $(\mathrm{p}=0.003)$, and suggestions $(\mathrm{p}=0.024)$.

\subsection{Implications}

While the subject pool in this preliminary study was admittedly small, the results strongly suggest a difference in the nature of verbal interactions between users in COPRESENT and REMOTE groups. For children sharing a single computer running the GSW, the experience was highly social, and conversation was nearly continuous. The shared nature of their task seemed obvious and users discussed their actions. A closer examination of the transcript, however, still shows little discussion of storytelling intentions or interpretation of events. The children at separate computers seemed to view their tasks as largely private, and initiated few interactions of any kind.

The following transcript of two children (one is five years old, the other six) working at a single computer shows examples of exchanges in several of the interaction categories. They ask questions regarding their collaborator's storytelling intent and about system events. They share interpretations of events. The students are obviously engaged in the storytelling task.

A: Want the cake?

B: OK. Pretend like the boy got the cake because he wasn't shy anymore.

Put the cake here. (points) You pressed the cake.

A: $\quad$ I'm going to put it behind the castle.

B: How come?

A: He's going to walk up here. (indicates boy moving to the new location of the cake)

(Selects girl character and places her in the illustration area)

The system displays the attribute menu

Shy? OK?

The system displays text introducing the girl; There was a shy girl. She wanted to make a friend.

B: $\quad$ Shy cause she wants a friend...

She said, "A-ha, I can go to the wishing well."

A: (Selects girl again)

B: $\quad$ Put it right next... (point to well)

A: $\quad$ (Places girl next to well)

The system displays text describing the move; The girl wanted to do something fun. She went to the well. The girl did a dance around the well.

B: What?

A: $\quad$ She's doing a dance.

B: How come she's dancing?

A: Because she's dancing for joy.

Unfortunately, such task specific interactions were very uncommon in the COPRESENT group and did not occur at all in the REMOTE group. Most discussions seemed to involve issues of protocol or observations of events.

While we expected to see some differences in the character of interactions between members of the REMOTE and COPRESENT groups, we did not expect the extremely low number of overall interactions between users in the REMOTE group. Previous work with collaborative computer learning environments had alerted us to some of the difficulties in encouraging verbal interactions, but we had expected that users in the REMOTE group would interact in order to coordinate their actions. We feel that there are several reasons why this interaction may not have occurred.

The novelty of the REMOTE setup may have been an issue. Some of the students in the study had experience working with stand- 
alone computers. They seemed comfortable with the notion of collaborating at the same machine. Collaborating at separate machines may be a more foreign concept requiring additional explanation or practice for users at this age. Students in the REMOTE group frequently looked over at their partner's display, as if to confirm that they really were seeing the same thing. The following exchange between two students is a good example of this confusion.

$$
\begin{array}{ll}
\text { A: } & \text { I see a person and a dog. } \\
\text { B: } & \text { So do I. } \\
\text { A: } & \text { Which one are you? } \\
\text { B: } & \text { This one. (points to screen) } \\
& \text { (to proctor) Now what do I do? }
\end{array}
$$

Also, the focus of each participant in the REMOTE group seemed to be toward building an individual story rather than a shared story with their partner. Such an observation is consistent with the experiences of other researchers. There was a large amount of simultaneous object movement and the users seemed hesitant to interrupt their partner with verbal interactions. The construction of the REMOTE activity with equivalent sets of objects initially placed in the staging area of each user may have encouraged these independent efforts. Users were given sufficient resources to explore a limited set storytelling states without interacting with their partner's objects. Though the collaborative opportunities were explained and demonstrated before the task, the possibility of achieving more complex story interactions (and the rewards of richer stories and additional interaction animations) through collaboration with their partner was apparently not obvious or sufficiently compelling.

\section{2D GSW INTERACTION STUDY 2}

In the Pilot study, not only did REMOTE users not engage in task-specific verbal interactions, they did not engage in many verbal interactions at all. To address these results, we decided to focus modifications to the GSW in two areas. The first concerned the distribution of resources. The multi-user software used in the Pilot Study distributed objects in an effort to provide equivalent sets to each user. This was done to create an environment for each user similar to that found in the stand-alone version of the software. By providing unique but inter-related sets of objects, we hoped to provide more incentive for verbal interactions.

The second area concerned goal assignment. In the Pilot Study, users were allowed to manually (and independently) select character goals. We felt that establishing a common initial goal would make the collaborative nature of the task more obvious and provide more reason to interact. In order to test these hypotheses, we performed another study with more students, and more time spent with the GSW.

\subsection{Method}

Twenty-six first-grade students participated in the study. The students were randomly assigned to pairs, which were then randomly assigned to one of three treatment groups. In one group (COPRESENT), pairs of students shared a single computer running the GSW. In the other two groups, pairs of students used the multi-user version of the GSW. Users in the first multi-user case (REMOTE-Same), each started their stories with identical sets of objects in their staging area. In the second multi-user case
(REMOTE-Unique), users were given differentiated sets of characters and objects. For example, if one user had a hungry dog, the other user had a bone. (Table 2) In all three cases, object characteristics were system generated, and the stories began by communicating a common goal or goals to all the users (e.g. "Once upon a time in a distant forest, there was a very hungry dog. He wished that he had something to eat.").

\begin{tabular}{|l|ll|ll|}
\hline \multirow{2}{*}{ COPRESENT } & \multicolumn{2}{|c|}{ REMOTE-Same } & \multicolumn{2}{|c|}{ REMOTE-Unique } \\
& User 1 & User 2 & User 1 & User 2 \\
\hline Bird & Bird & Bird & Bird & Dog \\
Nest & Nest & Nest & Bone & Nest \\
Dog & Dog & Dog & & \\
Bone & Bone & Bone & \\
\hline
\end{tabular}

Table 2: Sample Object Distribution

The children were trained in the use of the mouse and the operation of the Graphic Story Writer. They were then instructed to collaborate on three story scenarios of increasing complexity and given five minutes to work with each. The second and third scenarios presented opportunities for multiple conflict resolution (e.g. the hungry dog got his bone and the sleepy bird found a nest). If all the conflicts were resolved before the five minutes expired, students were allowed to continue playing with the system for the duration of their time. Audio and video records of the subjects' performances were collected, and transcripts of the verbal interactions (one per subject) were composed. The transcripts were coded by two independent scorers with strong agreement; the few discrepancies were resolved through negotiation.

\begin{tabular}{|ll|}
\hline PROTOCOL & $\begin{array}{l}\text { Discussion of turn taking (e.g. "You just } \\
\text { moved. Its my turn now."). }\end{array}$ \\
TASK & $\begin{array}{l}\text { Discussion and interpretation of story } \\
\text { events (e.g. "The hungry bear wants to } \\
\text { go and eat some honey."). }\end{array}$ \\
COORDINATE & $\begin{array}{l}\text { Discussion of observed or planned events } \\
\text { (e.g. "I see the dog moving."). }\end{array}$ \\
OTHER & All other utterances. \\
\hline
\end{tabular}

Table 3: Coding Scheme

The coding scheme was a modified version of the one used in the Pilot study (Table 3). Many of the questions and answers in the Pilot study related to rather simple, surface level details. We felt that it was important to relate interactions more closely to their intent. This resulted in four measures of verbal interaction: more substantive, task-related interactions (TASK), discussions of turn taking (PROTOCOL), discussions of surface level details (COORDINATE) off-task or miscellaneous interactions (OTHER), as well as the total number of verbal interactions. We also collected data on the total number of story interactions (MOVES), and whether the stories reflected a complete conflict resolution structure. 


\subsection{Results}

Analysis of variance was performed on the coded transcript data, using the total number of verbal interactions and the total interactions in each of the four categories. The total number of story events and the number of story conflicts resolved were also analyzed.

With this larger group of subjects, there was no significant difference between the groups in the total number of verbal interactions or interactions in the Protocol, Coordinate or Other categories. Likewise, there were no significant differences between the groups regarding the total number of story events generated or the total number of resolutions. Analysis of variance with repeated measures found no significant differences across the scenarios. Previous work has suggested gender differences in verbal interactions in CSCL settings, but analyzing the various measures according to gender produced no significant differences.

However, there was a significant difference in the amount of total task specific verbal interaction (TASK); $F(2,24)=6.251$, $\mathrm{p}=.0068$. Students in the REMOTE-UNIQUE group participated in more task specific interactions than students in both the COPRESENT and the REMOTE-SAME groups (Figure 9). Posthoc analysis with the Scheffe test reveals significant differences between the REMOTE-Unique group and both other groups: REMOTE-U > REMOTE-S = COPRESENT.

\begin{tabular}{|c|c|c|c|c|c|}
\hline & $\begin{array}{l}\text { Mean } \\
\text { REMOTE- } \\
\text { S }\end{array}$ & $\begin{array}{l}\text { Mean } \\
\text { REMOTE- } \\
\text { U }\end{array}$ & $\begin{array}{l}\text { Mean } \\
\text { COPRESENT }\end{array}$ & $F_{2,23}$ & $\mathbf{p}$ \\
\hline Resolutions & 2.4 & 2.25 & 2.75 & 0.911 & 0.416 \\
\hline Moves & 16.6 & 19.3 & 17.1 & 0.393 & 0.680 \\
\hline Total VI & 62 & 49 & 52 & 0.371 & 0.6942 \\
\hline Protocol & 3.3 & 1.8 & 11 & 3.032 & 0.0678 \\
\hline Task & 2 & 7 & 1 & 6.251 & 0.0068 \\
\hline Coordinate & 42 & 34 & 35 & 0.459 & 0.6378 \\
\hline Other & 14 & 6.9 & 5.9 & 1.669 & 0.2104 \\
\hline
\end{tabular}

Table 4: Results Across Scenarios

\subsection{Implications}

We were encouraged to see verbal interaction was nearly continuous between students in all the groups. Unlike the REMOTE group members in the Pilot Study, members of both REMOTE groups in this study engaged in amounts of verbal interaction similar to their counterparts in the COPRESENT group. No statistically significant difference was found for the total number of interactions between members of the three groups. We feel that the differences between these results and those in the Pilot study are likely due to the explicit introduction of common goals, and to examining multiple scenarios (3) over a longer period of time (15 minutes).

In all groups, the majority of interactions fell into the Coordinate category. These utterances served to inform partners of activity in progress or observed events. These interaction differed from Task Specific interactions in that they merely described actions or events, and did not involve story related inferences or planning. The following passage illustrates a Coordinating discussion:
A: What are you doing?
B: $\quad$ I just pressed the cat.
A: I'm trying to get a ball
B: $\quad$ I got the ball.
A: $\quad$ You're moving it?
B: Yup.

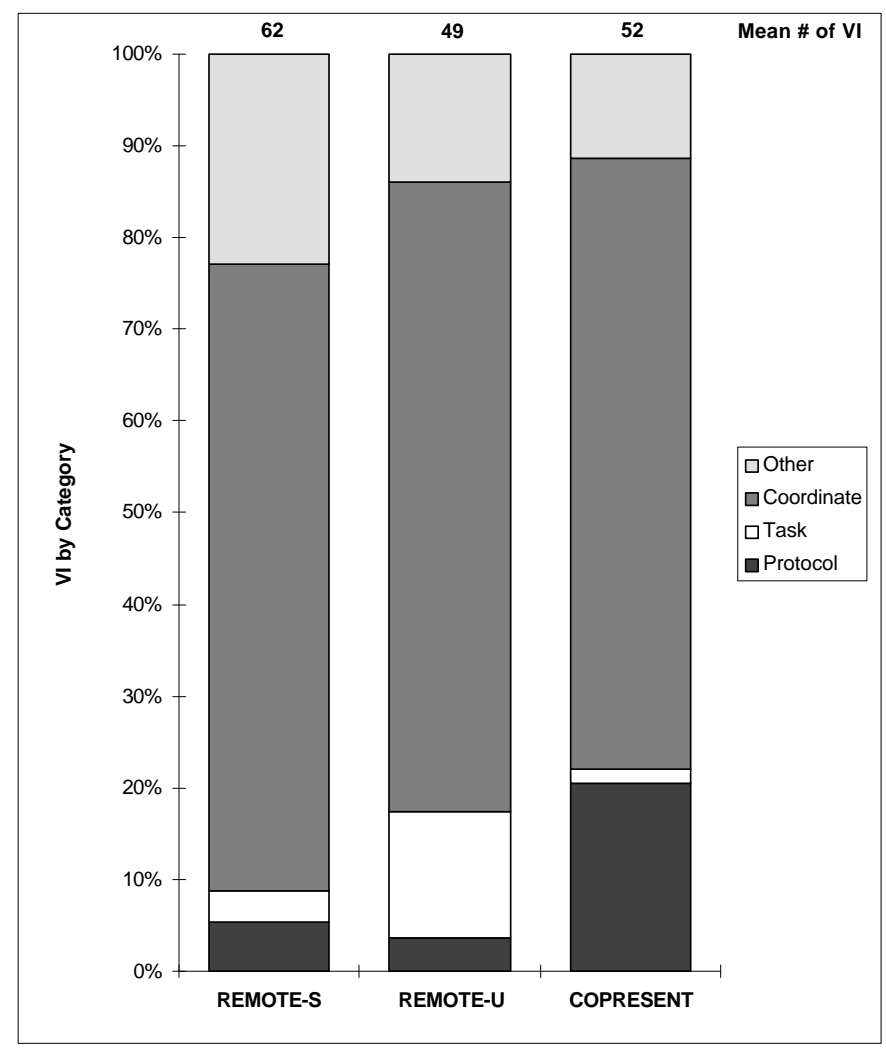

Figure 3: Mean Verbal Interaction

We hoped to see more than just an increase in total verbal interaction between students in the REMOTE groups; we also hoped to see more substantive discussion of their storytelling task, and this turned out to be the case. Students in the REMOTE-Same and REMOTE-Unique groups engaged in more Task Specific verbal interactions on the average (2.10 and 6.88 mean verbal interactions, respectively) than did members of the COPRESENT group (0.75 mean verbal interactions). While Task interactions were the least common type in the COPRESENT and REMOTESame groups, they were relatively more frequent in the REMOTEUnique group, scoring ahead of Protocol interactions. We believe that this result indicates an increase in attention to task rather than process.

The following is an example of Task specific interaction. Notice how the discussion involves elements or role-play (speaking for the characters) and problem solving (do sheep eat clover?), as opposed to mere descriptions of events as in the Coordinate interaction.
A:
(Moves bird to hungry sheep)
B: Come right here. The lamb wants to eat you. 
He said, "Hello, sheep. Give me food."

B: (Moves clover to sheep) I'm giving him food. Here's some plants to eat.

A: $\quad$ "I don't eat plants. I eat hay."

Protocol discussions were more common in the COPRESENT group than in the REMOTE groups, though statistical significance at $\mathrm{p}<.05$ was not reached on this measure. In almost all cases, students in both the COPRESENT and REMOTE groups decided to follow a turn taking protocol. Several extended protocol discussions in the COPRESENT pairs resulted from individuals who were reluctant to turnover control of the mouse to their partner. Although simultaneous activity was supported in the REMOTE groups, most users opted for asynchronous movements, and generally stuck to a turn-taking scheme.

These results have interesting implications for the design of realtime collaborative learning environments. For the subjects in our Pilot study, the side-by-side use of a shared workspace differed greatly in character from side-by-side use of a single machine. By attempting to reproduce the options available in the stand-alone version, our initial migration to a shared workspace did not maintain similar levels of verbal interaction. This indicates that, at least for some kinds of subjects and tasks, straightforward migration to shared environments may have unforeseen effects on communication patterns.

However, we were able to achieve positive changes in verbal interaction by clearly communicating a common goal and distributing resources. While differences in a shared workspace could be viewed as a liability in some circumstances, it could be a positive factor in collaborative learning settings, at least those in which discussion is desired. By communicating a common goal and by creating positive interdependences, we observed an increase in total interactions between shared workspace users and an increase in task specific verbal interactions.

\section{3D GRAPHIC STORY WRITER}

There is increasing interest in the educational use of virtual environments. Researchers cite a variety of benefits including enhanced motivation, support for visual learning styles, and facilitation of constructivist learning [18]. However, as with 2D shared workspaces, collaboration in these environments is not a forgone conclusion.

We felt that the introduction of positive interdependences could encourage collaboration in a $3 \mathrm{D}$ workspace as it had in a $2 \mathrm{D}$ workspace. In order to test this hypothesis, we are developing a $3 \mathrm{D}$ version of the GSW and report on our initial usability study and observations.

\subsection{D Design Differences}

In many respects, the 3D GSW is similar to the 2D GSW. Both tell simple stories appropriate to the target audience of Kindergarten and First Grade students. Both employ a similar narrative generation engine based on an identical story grammar. Both systems generate text as a result of explicit user interactions within the workspace. Both systems present a pre-defined set of characters, conflicts, and props.

However, several modifications to the interaction model needed to be made in order to support interaction in a $3 \mathrm{D}$ space. These include the size of the workspace, user point-of-view, interaction and navigation in the workspace, and support for positive interdependences.

Given the relatively small workspace of the 2D GSW, it was possible to present users with a view of the entire workspace. Users did not have to perform any explicit activities to control their view, and the workspaces of all participants showed identical views.

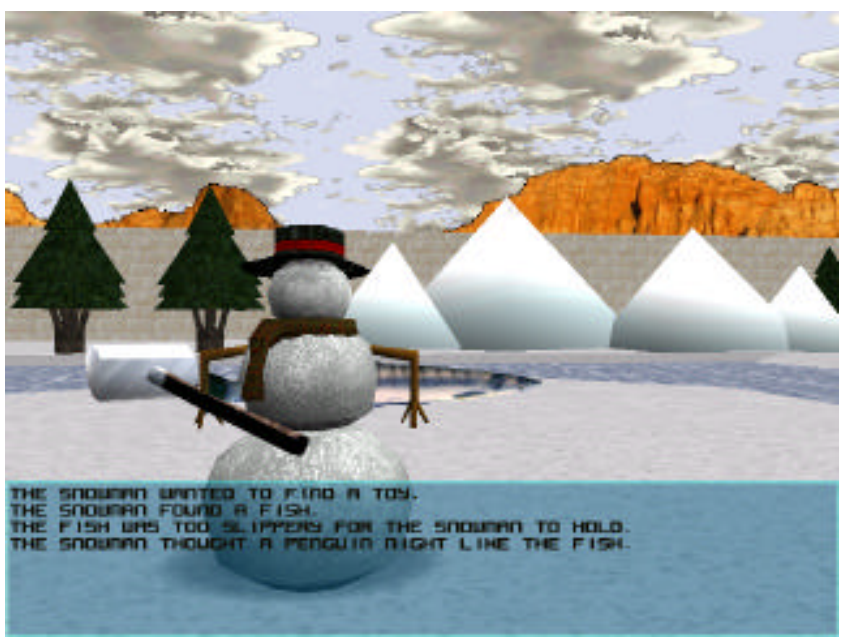

Figure 4: 3D Graphic Story Writer

Objects and characters in the 3D GSW were placed in an appropriately proportioned 3D setting (a fantasy snowscape of animated snowmen and playful penguins). Development of this $3 \mathrm{D}$ environment resulted in a richer, but larger, workspace. A number of design modifications were made in order support this more complex environment.

In the 3D GSW, it was no longer possible to view the entire workspace at a resolution that would also support object selection and manipulation. Rather than ask young users to assume control of arbitrarily positioned views or "cameras," we decided to provide users with a single view attached to a mobile avatar. While this change provided what would (hopefully) be an easy-tomanage means of controlling the user's view, it also changed the nature of the interaction in the GSW from that of an all-seeing author, to that of a situated avatar.

In the 2D GSW, users could select any of the available props or characters at any time and immediately place them (by clicking and dragging) anywhere in the workspace. While users in the 3D GSW still had the opportunity to interact with all objects in the environment, the nature of the interaction was modified to conform with the context established by the $3 \mathrm{D}$ environment and the situated avatar. Users were now limited to moving only their avatar and the props that it carried. In order to trigger an interaction with another character or prop, the user would need to navigate their avatar through the environment to the location of the desired character or prop, and initiate an interaction.

Lastly, the nature of the positive interdependences within the environment was slightly changed. Within the 2D GSW the positive interdependence was created by providing users with access to unique sets of props. With the 3D GSW, we created similar positive interdependences, but chose to limit the set of interactions supported by certain strategic props, rather than limit access to the prop entirely. 


\subsection{D GSW Usability Study}

Nine early elementary students participated in our usability study of the 3D GSW. Pairs of students were observed and videotaped using the 3D GSW together in a two-workstation side-by-side configuration similar to the physical arrangement described for the REMOTE conditions in the Verbal Interaction studies (one student participated in two sessions).

Sessions began with an introduction to the project, instruction in the basic controls (cursor keys for movement, spacebar for interaction), and an instruction to collaborate on a story. The subjects were asked to participate in at least one story, though all our test subjects elected to participate in at least two. The shortest session was 20 minutes, the longest was close to 60 and the average session for a pair of subjects was 30 minutes. After concluding work on one story, subjects were allowed to change workstations and experience the story from the viewpoint of the other character, and many elected to do so.

Our usability study hoped to uncover any issues in the interface design or interaction model that hindered use or collaboration. In particular, we wanted to observe how the design changes noted earlier (workspace size, user point of view, interaction model, positive interdependences) would impact the overall usability of the system, as well as how these changes would influence the amount and type of verbal interactions.

Overall, the users experienced no real difficulty with navigation within the 3D workspace. Most of the subjects had already had some experience navigating in similar 3D environments in $\mathrm{PC}$ or console games. The use of cursor controls for changing direction and moving forward and backwards was easily grasped and required no extra explanation (with one exception explained below). Similarly, users had little difficulty understanding the POV attached to the avatar.

One side effect of the introduction of the larger workspace was that movement of the avatar from one location to another in the 3D GSW required more time than the click-drag interactions of the 2D GSW. After receiving feedback from the early test sessions that the characters "moved too slowly," an accelerator was enabled for the later test sessions. While the accelerator allowed users to cover ground more rapidly, it also made fine movement more difficult. One user in particular had difficulty switching between rapid movement for covering long distances and slower movement for traversing difficult terrain (such as a bridge with no railings). Our intention was not to require that users master gamelike navigation, so our future designs will attempt to support more rapid movement while preventing accidents caused by the rapid movement (for instance, by providing a railing for the bridge). Another design alternative under consideration is reducing the distance between objects in the environment.

The process of moving the avatar to a desired location and initiating an interaction also presented no difficulties for our users. Some of the interactions that were significant to resolution of the characters goals were accompanied by animation and sound effects and the subjects expressed appreciation for these. The most common feedback we received from the subjects regarding interaction was that the environment should include more events that include animation and sound effects.

A number of user comments also related to consistency within the environment. As mentioned earlier, certain significant interactions would result in animations or changes to the state of characters. For example, interaction between the snowman and the hammer resulted in the snowman carrying the hammer. This activity was necessary in order to allow the snowman to remove an ice wall at a different location. Since picking up and using the hammer was necessary to the resolution of the goal we provided support for those interactions. We did not provide a corresponding ability to drop the hammer, since there was no goal-related need to remove it. However, several subjects mentioned that if it were possible to pick up objects, it should also be possible to drop them. Their desire for a consistent experience seemed in some ways just as important as having means for satisfying their characters goals.

Due perhaps to this same desire for a logically consistent experience, the model of positive interdependence in the 3D GSW seemed in some ways to be more acceptable and perceived as less restrictive to the target audience than the model presented in the 2D GSW. While some users expressed confusion by their inability to "see" necessary props in the 2D GSW, the context-appropriate explanations for why their characters could see but not access certain props in the 3D GSW was accepted with little confusion or frustration.

For example, in the 2D GSW, if a user was able to see a hungry penguin in his initial set of characters and props, he would not be able to see or manipulate the fish until the other user had introduced the fish into the workspace. While this arrangement did have the intended effect of encouraging discussions related to the characters and their goals, the means of achieving the effect was perceived by some as being somewhat restrictive.

In the 3D GSW however, more context-appropriate means were employed to achieve the same result. For example, a user might be given a hungry penguin as their avatar, but the fish would be placed on the other side of an ice wall that only the snowman was capable of breaking. While this approach had the same effect of creating positive interdependences between the characters, the consistent internal story logic seemed to make the restriction more acceptable to the participants.

A key usability goal for the 3D GSW was to maintain the levels and type of communication that were obtained by introducing positive interdependences (the REMOTE-UNIQUE condition) in the earlier study of the 2D GSW. While a more rigorous study of a larger set of test subjects will be necessary to confirm these findings, our initial observations indicate communication patterns consistent with the 2D GSW.

Students were aware of their characters goals, and after some exploration and initial attempts at satisfying their goals, became aware of the need to collaborate with their partners. All the subjects were able to resolve the conflicts posed for their characters which required intervention by their partner. While overall rates of communication related to coordination were high (as we observed in the prior studies), most pairs also engaged in task-related dialog as well - reviewing goals, discussing character attributes, and forming plans to satisfy the goals.

A: There you are. The shy penguin is over there and I'm trying to get him food.

B: (at ice wall) How do you get through this?

A: I don't know. It seems to be an ice thing.

B: Find the hammer. You... uh... have to break this thing. 
$\cdots$

A: How in the world am I supposed to get fish?

B: (moves toward fish) I think I may have something... lets see here... fish... Ahhh! Whoa. (almost falls in lake)... Alright. There's the fish. We need to find some sort of hammer.

The nature of interaction and communication changed over repeated sessions with the same story. In their initial session, subjects spent more time exploring and discussing plans for satisfying their character goals. Once the goals and the means for satisfying them had been established, dialog in subsequent sessions tended more towards coordination. Providing a new scenario for each session, or developing the capability to dynamically generate unique positive interdependences for each session might allow us to maintain the higher levels of taskrelated dialog observed in the initial sessions.

Another interesting observation was that once the students had satisfied the character goals for their session, they did not necessarily want to conclude their experience. Many exchanged workstations so that they could experience the story again from the point-of-view of the other character. And many began to form their own goals for interaction and exploration within the environment. One pair even set up their own game of hide-andseek within the environment.

\section{CONCLUSIONS}

In our studies, we found that creating positive interdependences between collaborators encouraged more task related dialog. While such task "problematization" could be viewed as undesirable in a conventional system, it proved to be a positive factor in our cooperative learning setting. Introducing positive interdependences in 2D and 3D shared workspaces seems a promising approach to encouraging discussion, and we hope this work can serve as starting point for continued investigation into this area.

Our work on the GSW has raised other intriguing research opportunities in the areas of narratives and learning. In addition to continued work on positive interdependences, we are interested in exploring the generation of richer narrative texts, alternative presentations of character motivation, and the dynamic generation and management of scenarios not only for learning about stories, but learning from stories.

\section{REFERENCES}

[1] Alborzi, Houman., et. al., Designing StoryRooms: interactive storytelling spaces for children, in Proceedings of DIS 2000 (NY, NT, August 2000), ACM Press, 95-104.

[2] Applebee, Arthur N., The Child's Concept of Story. University of Chicago Press, Chicago, 1978.

[3] Benford, Steve., et. al., Designing Storytelling Technologies to Encouraging Collaboration between Young Children, in Proceedings of ACM CHI 2000 Conference on Human Factors in Computing Systems 2000 v.1 p.556-563.

[4] Derry, Sharon J., Metacognitive Models of Learning and Instructional Systems Design, in M. Jones, P. Winne (Ed.) Adaptive Learning Environments. Springer-Verlag, NY, 1992, pp. 257-286.
[5] Johnson, David W. and Johnson, Roger T., Computer Assisted Cooperative Learning, Educational Technology, January 1986, pp. 12-18.

[6] Reiser, Brian J., Why Scoffolding Should Sometimes Make Tasks More Difficult for Learners, in Proceedings of CSCL 2002 (Boulder CO, January 2002), ACM Press, 255-264.

[7] Roussos, M., Johnson, A., Moher, T., Leigh, J., Vasilakis, C., and Barnes, C. (1998). Learning and Building Together in an Immersive Virtual World In Presence vol 8, no 3, June, 1999, MIT Press, pp. 247-263.

[8] Ryokai, Kimiko., Vaucelle, Catherine., Cassell, Justine., Literacy Learning by Storytelling with a Virtual Peer, in Proceedings of CSCL 2002 (Boulder CO, January 2002), ACM Press, 352-360.

[9] Rysavy S. Del Marie, Sales, Gregory C., Cooperative Learning in Computer-Based Instruction, Educational Technology Research and Development, v39(2), pp. 70-79.

[10] Scott, Stacey D., Mandryk, Regan L., Inkpen, Kori M., Understanding Children's Interactions in Synchronous Shared Environments, in Proceedings of CSCL 2002 (Boulder CO, January 2002), ACM Press, 333-341.

[11] Shuell, Thomas J., Designing Instructional Computing Systems for Meaningful Learning, in M. Jones, P. Winne (Ed.) Adaptive Learning Environments. Springer-Verlag, NY, 1992, pp. 19-54.

[12] Steiner, Karl E. and Moher, Thomas G. Graphic StoryWriter: An Interactive Environment for Emergent Storytelling. In Proceedings of CHI '92 (Monterey, CA, May 3-May 7). ACM , NY, 1992, pp. 357-363.

[13] Steiner, Karl E. and Moher, Thomas G. Scaffolding Story Construction with Interactive Multimedia, The Journal of Educational Multimedia and Hypermedia, vol 3 no 2, 1994, pp. 173-196.

[14] Sulzby, Elizabeth. Children's Development of Prosodic Distinctions in Telling and Dictation Modes, in Ann Matsuhashi (Ed.), Writing in Real Time. Ablex, Hillside, NJ, 1987.

[15] Vygotsky, L. Mind in society: The development of higher psychological processes. Cambridge, MA: Harvard University Press, 1978.

[16] Webb, N.M. Task related verbal interaction and mathematics learning in small groups. Journal for Research in Mathematics Education, 22 (5), 1991.

[17] Webb, N.M. Constructive Activity and Learning in Collaborative Small Groups. Journal of Educational Psychology, 87(3), 1995, pp. 406-423.

[18] Youngblut, C. Educational uses of virtual reality technology. Technical Report IDA Document D-2128, Institute for Defense Analyses, Alexandria, VA., 1998 